\title{
INTERIOR SPHERE PROPERTY OF ATTAINABLE SETS AND TIME OPTIMAL CONTROL PROBLEMS*
}

\author{
Piermarco CAnNARsA ${ }^{1}$ And HÉLÈne FrankowskA ${ }^{2}$
}

\begin{abstract}
This paper studies the attainable set at time $T>0$ for the control system$$
\dot{y}(t)=f(y(t), u(t)) \quad u(t) \in U
$$

showing that, under suitable assumptions on $f$, such a set satisfies a uniform interior sphere condition. The interior sphere property is then applied to recover a semiconcavity result for the value function of time optimal control problems with a general target, and to deduce $C^{1,1}$-regularity for boundaries of attainable sets.
\end{abstract}

Mathematics Subject Classification. 26B25, 49K15, 93B03.

Received April 20, 2004. Revised February 21, 2005.

\section{INTRODUCTION}

It is a fact quite commonly accepted that the structure of the attainable set (from 0 ) at time $T$ of the control system

$$
\left\{\begin{aligned}
\dot{y}(t) & =f(y(t), u(t)) & & (t>0) \\
u(t) & \in U & & \text { a.e. }
\end{aligned}\right.
$$

should be related to the geometry of the sets $\{f(x, U)\}_{x \in \mathbb{R}^{n}}$ of all admissible velocities. Obviously, the form that such a relationship can take varies from problem to problem. For instance, the attainable set - hereafter denoted by $\mathcal{A}(T)$ - is compact when all the sets $f(x, U)$ are convex and compact (see, e.g., [1]). Moreover, for linear systems of the form $f(x, u)=M x+L u, \mathcal{A}(T)$ is convex for every $T$. If, in addition, $L=I$ and $U$ is a convex body with a $C^{1}$-smooth boundary, then $\partial \mathcal{A}(T)$ is $C^{1}$-smooth as well, see [5].

This paper is a contribution to the analysis of the attainable sets of nonlinear control systems like (1). In particular, we are interested in detecting structural properties of the system which ensure that, for $T>0, \mathcal{A}(T)$ satisfies a uniform interior sphere condition. This last property is important from various points of view. For example, it gives a generalized upper bound for the boundary curvature of $\mathcal{A}(T)$. Moreover, it can be used to study the regularity of the Minimum Time function, as we will explain later on.

\footnotetext{
Keywords and phrases. Control theory, attainable sets, minimum time function, semiconcave functions.

* Work supported in part by the European Community's Human Potential Programme under contract HPRN-CT-2002-00281, [Evolution Equations].

1 Dipartimento di Matematica, Università di Roma Tor Vergata, Via della Ricerca Scientifica 1, 00133 Roma, Italy; cannarsa@mat.uniroma2.it

2 CREA, École Polytechnique, 1 Rue Descartes, 75005 Paris, France; franko@shs.polytechnique.fr
} 
In view of our initial remarks, it seems natural to conjecture that, in order for attainable sets to satisfy an interior sphere condition, the sets $f(x, U)$ should have the same property. In fact, the rigorous statement and proof of such a conjecture is the main result of this paper, see Theorem 3.5 and Corollary 3.8. It has to be underlined, however, that the above condition alone for $f(x, U)$ is not enough to obtain our result, since the $C^{1,1}$-smoothness of the map $x \mapsto f(x, u)$ also plays an important role. Indeed, in Section 4 we provide an example that shows that $\mathcal{A}(T)$ may not have the interior sphere property if $x \mapsto f(x, u)$ is just Lipschitz continuous.

An immediate - yet interesting - application of the above structural analysis, is the $C^{1,1}$-regularity of $\partial \mathcal{A}(T)$, for $T>0$ sufficiently small and $f(x, U) a$-regular, see Corollary 3.12. This result follows from a classical convexity criterion proved in [18].

Another application of the interior sphere property of attainable sets concerns the regularity of the value function of time optimal control problems with a general target. In such problems, as well-known, one seeks to minimize the time needed to steer the trajectory of system $(1)$, with the initial condition $y(0)=x$, to a given closed set $\mathcal{K} \subset \mathbb{R}^{n}$ called the target (see, e.g., [14]). The Minimum Time function $V_{\mathcal{K}}(x)$, defined as the value function of the above problem, turns out to be nonsmooth, and the maximal regularity one may expect is local semiconcavity in the complement of the target. Indeed, a semiconcavity result for $V_{\mathcal{K}}$ is derived in [8] (see also [7]) assuming that the target satisfies a uniform interior sphere condition, see Section 5.

In this paper, we extend the result of [8] to problems with a general target. Our method is straightforward: using the connection of the levet set $\mathcal{R}(\mathcal{K} ; t):=\left\{x \in \mathbb{R}^{n}: V_{\mathcal{K}}(x) \leq t\right\}$ with the attainable set at time $t$ for system (1) with $f$ replaced by $-f$, we can apply Theorem 3.5 to deduce that $\mathcal{R}(\mathcal{K} ; t)$ satisfies a uniform interior sphere condition. Then, the Minimum Time function with target $\mathcal{R}(\mathcal{K} ; t)$ is semiconcave in the complement of $\mathcal{R}(\mathcal{K} ; t)$, owing to the result of [8]. Since $V_{\mathcal{K}}$ coincides with such a function - up to the constant $t$ - the semiconcavity of $V_{\mathcal{K}}$ follows. We note that the semiconcavity of the value function of certain exit time problems has recently been obtained by Sinestrari [20] using a completely different approach, see Remark 5.5.

The outline of the paper is as follows. In Section 2 we have collected notations, basic definitions and preliminary results. In Section 3 we prove the interior sphere property, discussing the assumptions and some consequences of our results. Section 4 contains our counterexample. Section 5 is devoted to the analysis of the Minimum Time function.

\section{Preliminaries}

We denote by $\langle\cdot, \cdot\rangle$ and $|\cdot|$, respectively, the Euclidean scalar product and norm in $\mathbb{R}^{n}$. For any $n \times n$ matrix $M$, we denote by $M^{\star}$ the transpose of $M$ and by $\|M\|$ the usual operator norm of $M$, that is $\|M\|=\sup _{|x|=1}|M x|$.

For any $x \in \mathbb{R}^{n}$ and any $r>0$, we set $B(x, r)=\left\{y \in \mathbb{R}^{n}:|y-x|<r\right\}$, and we use the abbreviations $B(r)=B(0, r), B=B(1)$.

For any subset $S \subset \mathbb{R}^{n}$, we denote by $\bar{S}$ the closure of $S$, by $S^{\text {c }}=\mathbb{R}^{n} \backslash S$ the complement of $S$, by $\partial S$ the boundary of $S$, and by $\operatorname{int}(S)$ the interior of $S$. For any $\lambda>0$ and $z \in \mathbb{R}^{n}$, we set

$$
\lambda S=\left\{x \in \mathbb{R}^{n}: x=\lambda y, y \in S\right\}, \quad z+S=\left\{x \in \mathbb{R}^{n}: x=z+y, y \in S\right\} .
$$

So, $x+r B$ and $B(x, r)$ will be equivalent symbols for the ball of radius $r$ centered at $x$.

A well-known function that will be used in the sequel is the distance from $S$, that is

$$
d_{S}(x)=\inf _{y \in S}|y-x| \quad \forall x \in \mathbb{R}^{n}
$$

Another useful function, related to the one above, is the oriented boundary distance function, or, briefly, signed distance,

$$
b_{S}(x)=d_{S}(x)-d_{S^{\mathrm{c}}}(x) \quad \forall x \in \mathbb{R}^{n},
$$

whose properties have been analyzed in several papers (see, e.g., [12]). 
The contingent cone to $S$ at a point $x \in S$ is defined by

$$
T_{S}(x)=\left\{v \in \mathbb{R}^{n}: \liminf _{t \downarrow 0} \frac{d_{S}(x+t v)}{t}=0\right\} .
$$

The contingent cone can also be characterized as follows. A vector $v \in \mathbb{R}^{n}$ belongs to $T_{S}(x)$ if and only if there exist sequences $\left\{x_{i}\right\}$ in $S$ and $\left\{\lambda_{i}\right\}$ in $\mathbb{R}_{+}$such that $x_{i} \rightarrow x, \lambda_{i} \downarrow 0$, and

$$
\frac{x_{i}-x}{\lambda_{i}} \longrightarrow v \text {. }
$$

If $S$ is convex, then $T_{S}(x)$ is the closed cone spanned by $S-x$. Moreover, $v \in \operatorname{int}\left(T_{S}(x)\right)$ if and only if, for some $r>0, x+] 0, r] B(v, r) \subset \operatorname{int}(S)$ (see, e.g., [19]). This fact may be false when $S$ is not convex.

We denote by $N_{S}(x)$ the normal cone to $S$ at a point $x \in S$, defined as

$$
N_{S}(x)=\left\{p \in \mathbb{R}^{n}:\langle p, v\rangle \leq 0, \forall v \in T_{S}(x)\right\} .
$$

When $S$ is convex, $N_{S}(x)$ coincides with the normal cone of convex analysis.

Let now $S$ be closed, and let $r>0$.

Definition 2.1. We say that $S$ satisfies an interior sphere condition of radius $r$ at a point $x \in \partial S$ if $x$ belongs to some closed ball $y_{x}+r \bar{B} \subset S$. We say that $S$ satisfies an interior sphere condition of radius $r$, or that $S$ has the interior $r$-sphere property, if $S$ satisfies an interior sphere condition of radius $r$ at every point $x \in \partial S$. Finally, we say that $S$ satisfies a uniform interior sphere condition if $S$ has the interior $r$-sphere property for some $r>0$.

Notice that, if $S$ satisfies a uniform interior sphere condition, then it cannot possess "corners" pointing outwards (but it may well have inward pointing corners). In other terms, if $S$ has the interior $r$-sphere property, then its "boundary curvature" is bounded above by $1 / r$. It is also clear that, if $S$ has the interior $r$-sphere property, then $S$ also satisfies an interior sphere condition for any smaller radius $0<r^{\prime}<r$.

Example 2.2. A typical example of a set satisfying a uniform interior sphere condition is the ellipsoid

$$
\mathcal{E}_{M}=\left\{x \in \mathbb{R}^{n}:\langle M x, x\rangle \leq 1\right\}
$$

where $M$ is a positive definite symmetric $n \times n$ matrix. Denoting by $\lambda_{\min }$ and $\lambda_{\max }$ the smallest and the largest eigenvalue of $M$, respectively, it is easy to show that $\mathcal{E}_{M}$ satisfies an interior sphere condition for a suitable radius $r=r\left(\lambda_{\min }, \lambda_{\max }\right)>0$. Moreover, $r$ stays bounded away from 0 as long as neither $\lambda_{\min }$ goes to 0 nor $\lambda_{\max }$ to $\infty$.

Our next result provides a necessary condition for a set to satisfy an interior sphere condition, that will be used in the analysis of the counterexample of Section 4.

Proposition 2.3. Let $S$ be a closed convex subset of $\mathbb{R}^{n}$ and $x \in \partial S$ such that $S$ satisfies an interior sphere condition of radius $r>0$ at $x$. Then, $N_{S}(x)$ is a half-line and, letting $p$ be the outward unit normal to $S$ at $x$, we have:

$$
\forall y \in \partial S:\langle p, x-y\rangle \leq 2 r \quad \Longrightarrow \quad\langle p, x-y\rangle \leq \frac{1}{2 r}|x-y|^{2} .
$$

Proof. By assumption we have that $x \in \bar{B}_{r}\left(y_{x}\right) \subset S$ for some point $y_{x} \in S$. Then, $N_{S}(x)$ is a closed convex cone contained in $N_{\bar{B}_{r}\left(y_{x}\right)}(x)$, not reduced to $\{0\}$. Thus, $N_{S}(x)$ is a half-line.

Now, to check (4), let $y \in \partial S$ be such that $\langle p, x-y\rangle \leq 2 r$. Since $x-r p+r \bar{B} \subset S$, an elementary geometric argument (see Fig. 1) shows that $a:=x-\langle p, x-y\rangle p$ satisfies

$$
|y-a|^{2} \geq|u-a|^{2}=|x-a||v-a|=\langle p, x-y\rangle(2 r-\langle p, x-y\rangle) .
$$




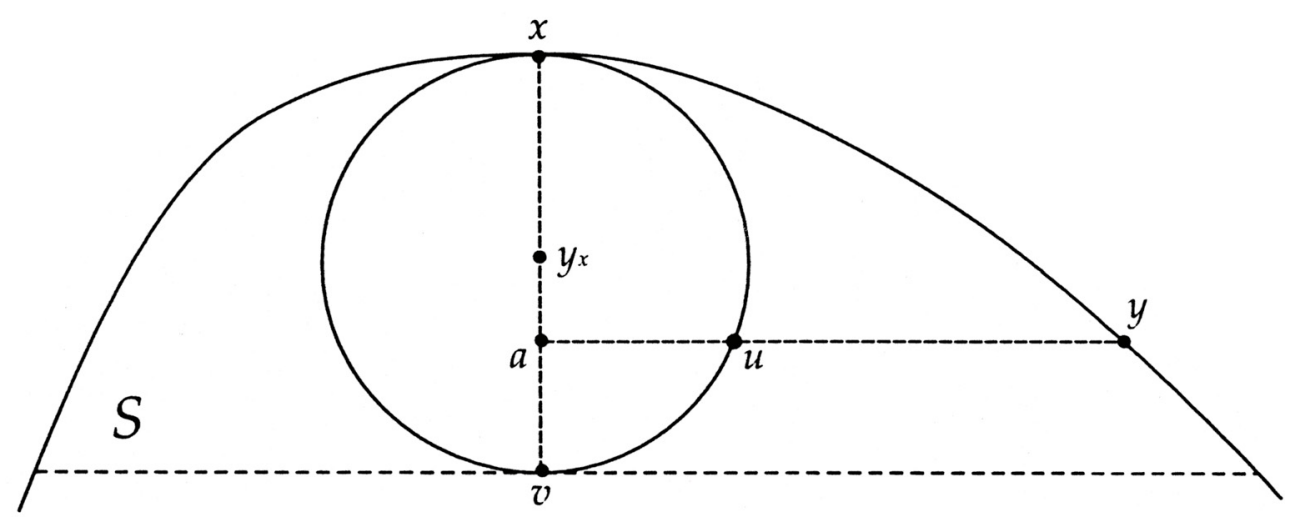

FiguRE 1. An elementary proof of (5).

Therefore,

$$
|x-y|^{2}=\langle p, x-y\rangle^{2}+|y-a|^{2} \geq 2 r\langle p, x-y\rangle
$$

as desired.

We conclude this section with a brief account of semiconcave functions. A function $\phi: \Omega \rightarrow \mathbb{R}$ is said to be semiconcave with linear modulus — or, briefly, semiconcave — in a subset $\Omega$ of $\mathbb{R}^{n}$ if there exists a constant $C \in \mathbb{R}$ (called a semiconcavity constant) such that $x \mapsto \phi(x)-C|x|^{2}$ is concave on every convex subset of $\Omega$. Any semiconcave function in $\Omega$ is also locally Lipschitz continuous in $\Omega$, since so are concave functions.

Whenever $\phi: \Omega \rightarrow \mathbb{R}$ is semiconcave on every compact set $Q \subset \Omega$, we say that $\phi$ is locally semiconcave in $\Omega$. Needless to say, in this case the semiconcavity constant $C_{Q}$ may blow up to $+\infty$ as $Q$ approaches the boundary of $\Omega$.

A function $\phi$ is said lo be semiconvex if $-\phi$ is semiconcave. It is a fact that $\phi$ is both semiconcave and semiconvex in $\bar{\Omega}$ if and only if $\phi \in C^{1,1}(\bar{\Omega})$.

The class of semiconcave functions plays an important role in nonsmooth analysis, control theory, and partial differential equations, see [9] for a comprehensive exposition of this theory.

\section{THE INTERIOR SPHERE PROPERTY OF ATTAINABLE SETS}

In this section we derive the main results of the paper. The exposition is organized in subsections in order to achieve a more efficient way of presenting the necessary arguments and computations. First, we present the standing hypotheses needed in the sequel, describe some of their consequences, and recall classical results for later use. In Section 3.2, we state and prove our main results, for which we have to introduce an additional assumption that we discuss later, in Section 3.3. Finally, in Section 3.4, we apply the previous theory to obtain a regularity result for the boundaries of attainable sets.

\subsection{Set-up}

Let a compact set $U \subset \mathbb{R}^{m}, m \geq 1$, and a map $f: \mathbb{R}^{n} \times U \rightarrow \mathbb{R}^{n}$ be given. The set

$$
F(x):=f(x, U)
$$

will be referred to as the set of admissible velocities at $x \in \mathbb{R}^{n}$. The standing hypotheses of this paper are the following. 


\section{Assumptions (A):}

(A0) $F(x)$ is convex for every $x \in \mathbb{R}^{n}$;

(A1) $f$ is continuous and $\exists L_{0}>0$ such that

$$
|f(x, u)-f(y, u)| \leq L_{0}|x-y| \quad \forall x, y \in \mathbb{R}^{n}, \forall u \in U ;
$$

(A2) $f(\cdot, u)$ is differentiable for every $u \in U$, and $\exists L_{1}>0$ such that

$$
\left\|D_{x} f(x, u)-D_{x} f(y, u)\right\| \leq L_{1}|x-y| \quad \forall x, y \in \mathbb{R}^{n}, \forall u \in U,
$$

where $D_{x} f$ denotes the Jacobian matrix of $f(x, u)$ with respect to $x$;

(A3) there exist an open set $\mathcal{O} \subset \mathbb{R}^{n}$ and a number $r>0$ such that, for every $x \in \mathcal{O}, F(x)$ satisfies an interior sphere condition of radius $r$.

Assumptions (A0), (A1) and (A2) are standard in control thery. They have been imposed on the whole space $\mathbb{R}^{n}$ just for simplicity. For the results of interest to this paper, they can be required - like (A3) - only on a given open set $\mathcal{O} \subset \mathbb{R}^{n}$.

Under assumptions (A0) and (A1), for all $x \in \mathbb{R}^{n}$, all $T>0$, and every measurable function $u:[0, T] \rightarrow U$ (usually called an admissible control), the Cauchy problem

$$
\left\{\begin{array}{l}
\dot{y}(t)=f(y(t), u(t)) \\
y(0)=x
\end{array}\right.
$$

has a unique solution in $[0, T]$, that will be hereafter denoted by $y(\cdot ; x, u)$. The role of assumption (A2) is to ensure that $y(\cdot ; x, u)$ is differentiable with respect to $x$.

The geometric assumption (A3) will be crucial for our analysis. It guarantees that the velocity set $F(x)$ is $n$-dimensional, and, together with the convexity assumption (A0), ensures that its (topological) boundary, $\partial F(x)$, is of class $C^{1,1}$.

Further consequences of the above assumptions are discussed in the proposition below. We recall that $b_{F(x)}$ stands for the oriented distance defined in (2).

Proposition 3.1. Assume (A0) and (A1). Then, for all $x, y \in \mathbb{R}^{n}$,

$$
v \in \partial F(x) \quad \Longrightarrow \quad\left|b_{F(y)}(v)\right| \leq L_{0}|x-y| .
$$

If, in addition, (A3) is satisfied, then, for all $x \in \mathcal{O}$, the following holds:

(a) the map $v \mapsto b_{F(x)}(v)$ is of class $C^{1,1}$ on $\partial F(x)+\frac{r}{2} B,\left|\nabla b_{F(x)}\right|=1$ on such a set, and

$$
\left|\nabla b_{F(x)}(v)-\nabla b_{F(x)}\left(v^{\prime}\right)\right| \leq \frac{2}{r}\left|v-v^{\prime}\right| \quad \forall v, v^{\prime} \in \partial F(x)+\frac{r}{2} B
$$

(b) for every $v \in \partial F(x), \nabla b_{F(x)}(v)$ is the outward unit normal to $F(x)$ at $v$ and

$$
v-r \nabla b_{F(x)}(v)+r \bar{B} \subset F(x)
$$

(c) for every $v \in\left(\partial F(x)+\frac{r}{2} B\right) \cap F(x)$, we have $v-\frac{r}{2} \nabla b_{F(x)}(v)+\frac{r}{2} \bar{B} \subset F(x)$.

Proof. To derive (9), let $v:=f(x, u)$. Suppose, first, $v \notin F(y)$. Then,

$$
0 \leq b_{F(y)}(v) \leq|f(y, u)-f(x, u)| \leq L_{0}|x-y| .
$$


Suppose now $v \in F(y)$, and fix a unit vector $p$ in the normal cone to the convex set $F(x)$ at $v$. Then, for all $v^{\prime}=f\left(y, u^{\prime}\right) \in F(y)$,

$$
\left\langle p, v^{\prime}-v\right\rangle=\left\langle p, f\left(y, u^{\prime}\right)-f\left(x, u^{\prime}\right)\right\rangle+\left\langle p, f\left(x, u^{\prime}\right)-f(x, u)\right\rangle \leq L_{0}|x-y| .
$$

Let $\bar{t}:=\max \{t \geq 0: v+t p \in F(y)\}$. Then, $\bar{t}$ is an upper bound for the distance of $v$ from $F(y)^{\mathrm{c}}$. So, taking $v^{\prime}=v+\bar{t} p$ we have

Inequality (9) follows from (12) and (13).

$$
\left|b_{F(y)}(v)\right| \leq \bar{t} \leq L_{0}|x-y|
$$

Now, assume (A3). Then, the fact that the signed distance is smooth near $\partial F(x)$ is well-known. We give a proof for the reader's convenience. For all $x \in \mathcal{O}, d_{F(x)}$ is convex and semiconcave on $F(x)^{\mathrm{c}}$, with semiconcavity constant equal to $1 / r$, see, e.g., [9], Proposition 2.2.2. Hence, $d_{F(x)}$ is of class $C^{1,1}$ up to the boundary of $F(x)^{\mathrm{c}}$. Similarly, $d_{F(x)^{\mathrm{c}}}$ is concave on $F(x)$. Moreover, the set $F_{r}(x):=F(x) \backslash\left(\partial F(x)+\frac{r}{2} B\right)$ also satisfies a uniform interior sphere condition of radius $r / 2$ by construction. So, $d_{F_{r}(x)}$ is semiconcave on $F_{r}(x)^{\mathrm{c}}$ with semiconcavity constant equal to $2 / r$. Since $d_{F(x)^{\mathrm{c}}}(v)+d_{F_{r}(x)}(v)=r / 2$ for every $v \in\left(\partial F(x)+\frac{r}{2} B\right) \cap F(x), d_{F(x)^{\mathrm{c}}}$ is semiconvex on $\left(\partial F(x)+\frac{r}{2} B\right) \cap F(x)$ with semiconvexity constant equal to $2 / r$. Therefore, $d_{F(x)^{\text {c }}}$ is of class $C^{1,1}$ on such a set. Since the gradients of $d_{F(x)^{\mathrm{c}}}$ and $-d_{F(x)}$ agree on $\partial F(x), b_{F(x)}=d_{F(x)}-d_{F(x)^{\mathrm{c}}}$ is continuously differentiable on $\left(\partial F(x)+\frac{r}{2} B\right) \cup F(x)^{\mathrm{c}}$, and $\nabla b_{F(x)}$ is a unit vector satisfying (10).

Point (b) is clear: (11) follows from (A3).

Finally, to prove (c), observe that $\bar{v}=v-b_{F(x)}(v) \nabla b_{F(x)}(v)$ is the projection of $v$ onto $\partial F(x)$. Moreover, $\nabla b_{F(x)}(\bar{v})=\nabla b_{F(x)}(v)$. Then,

$$
v-\frac{r}{2} \nabla b_{F(x)}(v)+\frac{r}{2} \bar{B} \subset \bar{v}-r \nabla b_{F(x)}(\bar{v})+r \bar{B} \subset F(x),
$$

thanks to $(11)$.

Let $\mathcal{K} \subset \mathbb{R}^{n}$ be a closed set. We introduce below the object of our analysis, that is the notion of attainable set for system (8). Let $t \geq 0$ be given.

Definition 3.2. The attainable set from $\mathcal{K}$ at time $t, \mathcal{A}(\mathcal{K} ; t)$, is the set of all points $y(t ; x, u)$, where $x \in \mathcal{K}$ and $u(\cdot)$ is an admissible control.

The set $\mathcal{A}(\mathcal{K} ; t)$ is also referred to as the reachable set, or the accessibility set, from $\mathcal{K}$ at time $t$. It is well-known that $\mathcal{A}(\mathcal{K} ; t)$ is closed for all $t \geq 0$ (see, e.g. [1]).

We will first analyze the special case $\mathcal{K}=\{0\}$, abbreviating notations as follows:

$$
y(\cdot ; u)=y(\cdot ; 0, u), \quad \mathcal{A}(\{0\} ; t)=\mathcal{A}(t) .
$$

It is easy to see that $\mathcal{A}(t)$ is bounded for every $t \geq 0$. Indeed,

$$
|f(x, u)| \leq|f(0, u)|+L_{0}|x| \leq H_{0}+L_{0}|x| \quad \forall(x, u) \in \mathbb{R}^{n} \times U,
$$

where

$$
H_{0}:=\max _{u \in U}|f(0, u)|
$$

(hereafter, we shall assume $H_{0}>0$, otherwise $\mathcal{A}(t)=\{0\}$ ). Then, for every $t \geq 0$ and every control $u:[0, t] \rightarrow U$,

$$
|y(t ; u)| \leq \frac{H_{0}}{L_{0}}\left(\mathrm{e}^{L_{0} t}-1\right)
$$

and so $\mathcal{A}(t)$ is bounded. More precisely, for any $R>0$ there exists $T_{R}>0$ such that $\mathcal{A}(t)$ is contained in $\bar{B}(R)$ for all real numbers $t \in\left[0, T_{R}\right]$, a possible choice of $T_{R}$ being

$$
T_{R}:=\frac{1}{L_{0}} \log \left(1+\frac{L_{0} R}{H_{0}}\right) .
$$


Now, let $x \in \partial \mathcal{A}(T)$ for a given $T \geq 0$. Since $\mathcal{A}(T)$ is closed, $x$ is the endpoint of a trajectory, i.e., $x=y(T ; u)$ for some admissible control $u(\cdot)$. Such a trajectory is said to be a boundary trajectory for system (8) for one can prove that $y(t ; u) \in \partial \mathcal{A}(t)$ for every $t \in[0, T]$. Boundary trajectories satisfy the Pontryagin Maximum Principle below (see, for instance, $[10,15]$, or $[13]$ ).

Theorem 3.3. Let $y_{0}(\cdot)=y\left(\cdot ; u_{0}\right)$ be a boundary trajectory for system (8), associated with a control $u_{0}:[0, T] \rightarrow$ $U$. Then, there exists an absolutely continuous function $p_{0}$, with $p_{0}(t) \neq 0$ for every $t \in[0, T]$, satisfying the adjoint system

and the maximum principle

$$
-\dot{p}(t)=\left(D_{x} f\right)^{\star}\left(y_{0}(t), u_{0}(t)\right) p(t)
$$

$$
\max _{u \in U}\left\langle p(t), f\left(y_{0}(t), u\right)\right\rangle=\left\langle p(t), f\left(y_{0}(t), u_{0}(t)\right)\right\rangle
$$

for a.e. $t \in[0, T]$.

\subsection{Main results}

Our analysis of attainable sets requires assumptions (A) described in the previous section, as well as an additional smoothness property of the set-valued map $F(x)=f(x, U)$ which we introduce in the definition below. Let $r$ and $L_{0}$ be the constants appearing in (A).

Definition 3.4. We say that $x \rightsquigarrow \partial F(x)$ is a Lipschitz boundary map if, for certain real numbers $r_{0} \in\left(0, \frac{r}{2 L_{0}}\right]$ and $C_{0} \geq 0$,

$$
\left|\nabla b_{F(y)}(v)-\nabla b_{F(x)}(v)\right| \leq C_{0}|x-y|
$$

for all $x \in \mathcal{O}$, all $y \in \bar{B}\left(x, r_{0}\right) \cap \mathcal{O}$, and all $v \in \partial F(x)$.

Notice that, in (19), $v$ turns out to be in $\partial F(y)+\frac{r}{2} B$ in view of (9). So, owing to Proposition 3.1(a), $\nabla b_{F(y)}(v)$ exists and is equal to the outward unit normal to $F(y)$ at the projection of $v$ onto $\partial F(y)$. The above notion will be further discussed in Section 3.3.

We are now ready for our first main result.

Theorem 3.5. Under assumptions $(\mathrm{A})$, suppose $0 \in \mathcal{O}$ and let $R>0$ be such that $R \bar{B} \subset \mathcal{O}$. If $x \rightsquigarrow \partial F(x)$ is a Lipschitz boundary map, then, for every $T \in\left(0, T_{R}\right)^{1}$, there exists $\rho_{T}>0$ such that $\mathcal{A}(T)$ satisfies an interior sphere condition of radius $\rho_{T}$.

Proof. To begin with, let $0<T<T_{R}$, let $z_{0}=y\left(T ; u_{0}\right)$ be a boundary point of $\mathcal{A}(T)$, and denote by $y_{0}(\cdot):=y\left(\cdot ; u_{0}\right)$ the corresponding boundary trajectory. Then, Theorem 3.3 guarantees the existence of an absolutely continuous function $p_{0}$, with $p_{0}(t) \neq 0$ for every $t \in[0, T]$, satisfying the adjoint system (17) and the Maximum Principle (18). In particular, from the last property, it follows that $v_{0}(t):=f\left(y_{0}(t), u_{0}(t)\right)$ stays on $\partial F\left(y_{0}(t)\right)$ for a.e. $t \in[0, T]$, and $p_{0}(t)$ is an outward normal vector to $F\left(y_{0}(t)\right)$. So,

$$
\nabla b_{F\left(y_{0}(t)\right)}\left(v_{0}(t)\right)=\frac{p_{0}(t)}{\left|p_{0}(t)\right|} \quad \text { for a.e. } t \in[0, T] .
$$

Moreover, $p_{0}$ can be normalized so that $\theta_{0}:=p_{0}(T)$ is a unit vector. Then,

$$
p_{0}(t)=P^{\star}(T, t) \theta_{0} \quad \forall t \in[0, T],
$$

where $P(t, s)$, defined for $t, s \in[0, T]$, is the matrix solution of

$$
\left\{\begin{array}{l}
\frac{\partial P}{\partial t}(t, s)=D_{x} f\left(y_{0}(t), u_{0}(t)\right) P(t, s) \\
P(s, s)=I
\end{array}\right.
$$

\footnotetext{
${ }^{1} T_{R}$ is defined in (16).
} 
Clearly,

$$
\|P(t, s)\| \leq \mathrm{e}^{L_{0}|t-s|} \quad \forall s, t \in[0, T] .
$$

Now, for any vector $\theta \in \bar{B}$, let us set

$$
y_{\theta}(t)=y_{0}(t)-\tau t P(t, T)\left(\theta_{0}-\theta\right) \quad \forall t \in[0, T],
$$

where $\tau>0$ is fixed so that $y_{\theta}(t) \in B\left(y_{0}(t), \frac{r_{0}}{2}\right) \cap R \bar{B}$ for all $t \in[0, T]$, and $r_{0} \in\left(0, \frac{r}{2 L_{0}}\right]$ is the radius provided by Definition 3.4. For this, in view of (15), it suffices to satisfy the following inequalities:

$$
2 \tau T \mathrm{e}^{L_{0} T} \leq \frac{r_{0}}{2}, \quad 2 \tau T \mathrm{e}^{L_{0} T}+\frac{H_{0}}{L_{0}}\left(\mathrm{e}^{L_{0} T}-1\right) \leq R
$$

Notice that $R-\frac{H_{0}}{L_{0}}\left(e^{L_{0} T}-1\right)>0$ since $T<T_{R}$.

Next, observe that

$$
z_{0}=y_{\theta_{0}}(T) \in\left\{y_{\theta}(T): \theta \in \bar{B}\right\}=z_{0}-\tau T \theta_{0}+\tau T \bar{B}
$$

Moreover, $y_{\theta}(0)=0$. So, in order to show that $\mathcal{A}(T)$ satisfies an interior sphere condition of radius $\tau T$ at $z_{0}$, it suffices to prove that, for every $\theta \in \bar{B}, y_{\theta}$ is a solution of $\dot{y}=f(y, u)$ for some control $u(\cdot)$.

Aiming at this, let us set $v_{\theta}(t)=f\left(y_{\theta}(t), u_{0}(t)\right)$. We claim that

$$
v_{\theta}(t) \in\left(\partial F\left(y_{\theta}(t)\right)+\frac{r}{2} B\right) \cap F\left(y_{\theta}(t)\right) \text { for a.e. } t \in[0, T] .
$$

Indeed, since $y_{\theta}(t) \in B\left(y_{0}(t), \frac{r_{0}}{2}\right)$ and $r_{0} \leq \frac{r}{2 L_{0}}$, we have that

$$
v_{0}(t) \in \partial F\left(y_{\theta}(t)\right)+\frac{r}{4} B \quad \text { for a.e. } \quad t \in[0, T]
$$

according to (9). Moreover, $\left|v_{\theta}(t)-v_{0}(t)\right|<\frac{r}{4}$ due to (A1) and the choice of $r_{0}$, whence the conclusion (25).

Then, in view of Proposition 3.1(a), we can represent the derivative $\dot{y}_{\theta}$ as

$$
\dot{y}_{\theta}(t)=v_{\theta}(t)-\frac{r}{2} \nabla b_{F\left(y_{\theta}(t)\right)}\left(v_{\theta}(t)\right)+\phi(t)+\frac{r}{2} w(t) \quad \text { for a.e. } \quad t \in[0, T]
$$

where

and

$$
\phi(t):=f\left(y_{0}(t), u_{0}(t)\right)-f\left(y_{\theta}(t), u_{0}(t)\right)-\tau t D_{x} f\left(y_{0}(t), u_{0}(t)\right) P(t, T)\left(\theta_{0}-\theta\right)
$$

$$
w(t):=\nabla b_{F\left(y_{\theta}(t)\right)}\left(v_{\theta}(t)\right)-\frac{2 \tau}{r} P(t, T)\left(\theta_{0}-\theta\right) .
$$

The idea of the proof consists in applying Proposition 3.1(c) to the right-hand side of (26), to conclude that $\dot{y}_{\theta}(t) \in F\left(y_{\theta}(t)\right)$. For this, we will have to show that $\phi(t)+\frac{r}{2} w(t) \in \frac{r}{2} \bar{B}$. Now, an easy computation based on (7) yields

$$
|\phi(t)| \leq L_{1}\left|y_{0}(t)-y_{\theta}(t)\right|^{2} \leq L_{1} \tau^{2} t^{2} \mathrm{e}^{2 L_{0}(T-t)}\left|\theta_{0}-\theta\right|^{2} \quad \forall t \in[0, T]
$$

So, let us proceed to estimate $w$. Recalling (20) and (21), we obtain

$$
\begin{aligned}
|w(t)|^{2}= & 1-\frac{4 \tau}{r}\left\langle P(t, T)\left(\theta_{0}-\theta\right), \nabla b_{F\left(y_{\theta}(t)\right)}\left(v_{\theta}(t)\right)\right\rangle+\left(\frac{2 \tau}{r}\right)^{2}\left|P(t, T)\left(\theta_{0}-\theta\right)\right|^{2} \\
= & 1-\frac{4 \tau}{r\left|p_{0}(t)\right|}\left\langle P(t, T)\left(\theta_{0}-\theta\right), P^{\star}(T, t) \theta_{0}\right\rangle+\left(\frac{2 \tau}{r}\right)^{2}\left|P(t, T)\left(\theta_{0}-\theta\right)\right|^{2} \\
& -\frac{4 \tau}{r}\left\langle P(t, T)\left(\theta_{0}-\theta\right), \nabla b_{F\left(y_{\theta}(t)\right)}\left(v_{\theta}(t)\right)-\nabla b_{F\left(y_{0}(t)\right)}\left(v_{0}(t)\right)\right\rangle .
\end{aligned}
$$


Moreover, since $|\theta| \leq 1=\left|\theta_{0}\right|$,

$$
\left\langle P(t, T)\left(\theta_{0}-\theta\right), P^{\star}(T, t) \theta_{0}\right\rangle=\left\langle\theta_{0}-\theta, \theta_{0}\right\rangle=\frac{1}{2}\left(\left|\theta_{0}\right|^{2}-|\theta|^{2}+\left|\theta_{0}-\theta\right|^{2}\right) \geq \frac{1}{2}\left|\theta_{0}-\theta\right|^{2} .
$$

Furthermore, to bound the last term in (28) we note that

$$
\begin{aligned}
& \left|\nabla b_{F\left(y_{\theta}(t)\right)}\left(v_{\theta}(t)\right)-\nabla b_{F\left(y_{0}(t)\right)}\left(v_{0}(t)\right)\right| \\
& \quad \leq\left|\nabla b_{F\left(y_{\theta}(t)\right)}\left(v_{\theta}(t)\right)-\nabla b_{F\left(y_{\theta}(t)\right)}\left(v_{0}(t)\right)\right|+\left|\nabla b_{F\left(y_{\theta}(t)\right)}\left(v_{0}(t)\right)-\nabla b_{F\left(y_{0}(t)\right)}\left(v_{0}(t)\right)\right| \\
& \quad \leq \frac{2}{r}\left|v_{\theta}(t)-v_{0}(t)\right|+C_{0}\left|y_{\theta}(t)-y_{0}(t)\right|
\end{aligned}
$$

using (10) since $v_{0}(t), v_{\theta}(t) \in \partial F\left(y_{\theta}(t)\right)+\frac{r}{2} B$, and using (19) since $v_{0}(t) \in \partial F\left(y_{0}(t)\right)$. Therefore, in view of (22),

$$
\left|\nabla b_{F\left(y_{\theta}(t)\right)}\left(v_{\theta}(t)\right)-\nabla b_{F\left(y_{0}(t)\right)}\left(v_{0}(t)\right)\right| \leq\left(\frac{2 L_{0}}{r}+C_{0}\right) \tau t \mathrm{e}^{L_{0}(T-t)}\left|\theta_{0}-\theta\right| .
$$

Then,

$$
\left|\left\langle P(t, T)\left(\theta_{0}-\theta\right), \nabla b_{F\left(y_{\theta}(t)\right)}\left(v_{\theta}(t)\right)-\nabla b_{F\left(y_{0}(t)\right)}\left(v_{0}(t)\right)\right\rangle\right| \leq C_{1} \tau t \mathrm{e}^{2 L_{0}(T-t)}\left|\theta_{0}-\theta\right|^{2},
$$

where $C_{1}:=\frac{2 L_{0}}{r}+C_{0}$. Therefore, again by $(22)$,

$$
\begin{aligned}
|w(t)|^{2} & \leq 1-\frac{2 \tau}{r\left|p_{0}(t)\right|}\left|\theta_{0}-\theta\right|^{2}+\left(\frac{2 \tau}{r}\right)^{2} \mathrm{e}^{2 L_{0}(T-t)}\left|\theta_{0}-\theta\right|^{2}+\frac{4 \tau^{2}}{r} t C_{1} \mathrm{e}^{2 L_{0}(T-t)}\left|\theta_{0}-\theta\right|^{2} \\
& \leq 1-\frac{2 \tau}{r} \mathrm{e}^{-L_{0}(T-t)}\left\{1-\frac{2 \tau}{r} \mathrm{e}^{3 L_{0}(T-t)}\left(1+r t C_{1}\right)\right\}\left|\theta_{0}-\theta\right|^{2} .
\end{aligned}
$$

Thus, using the elementary inequality $\sqrt{1+\alpha} \leq 1+\alpha / 2$, we conclude that

$$
|w(t)| \leq 1-\frac{\tau}{r} \mathrm{e}^{-L_{0}(T-t)}\left\{1-\frac{2 \tau}{r} \mathrm{e}^{3 L_{0}(T-t)}\left(1+r t C_{1}\right)\right\}\left|\theta_{0}-\theta\right|^{2} \quad \forall t \in[0, T] .
$$

Next, combine estimates (27) and (29), to derive

$$
\left|\phi(t)+\frac{r}{2} w(t)\right| \leq \frac{r}{2}-\frac{\tau}{2} \mathrm{e}^{-L_{0}(T-t)}\left\{1-2 \tau \mathrm{e}^{3 L_{0}(T-t)}\left(\frac{1}{r}+C_{1} t+L_{1} t^{2}\right)\right\}\left|\theta_{0}-\theta\right|^{2} \quad \forall t \in[0, T] .
$$

Now, possibly reducing $\tau$ in order to have

$$
1-2 \tau \mathrm{e}^{3 L_{0}(T-t)}\left(\frac{1}{r}+C_{1} t+L_{1} t^{2}\right) \geq 0 \quad \forall t \in[0, T],
$$

we obtain $\left|\phi(t)+\frac{r}{2} w(t)\right| \leq \frac{r}{2}$ for every $t \in[0, T]$. So, $\dot{y}_{\theta}(t) \in F\left(y_{\theta}(t)\right)$ owing to (26) and Proposition 3.1(c). Then, the Measurable Selection theorem (see, e.g., [2]) implies that $y_{\theta}(\cdot)$ is a trajectory of (8) with $x=0$.

Remark 3.6. From the above proof we obtain an estimate for $\rho_{T}$. Indeed, since $\rho_{T}=\tau T$ and $\tau$ must satisfy (24) and (30) with $C_{1}:=\frac{2 L_{0}}{r}+C_{0}$, one can take

$$
\rho_{T}=\frac{\mathrm{e}^{-L_{0} T}}{2} \min \left\{\frac{r_{0}}{2}, R-\frac{H_{0}}{L_{0}}\left(\mathrm{e}^{L_{0} T}-1\right), \frac{r T \mathrm{e}^{-2 L_{0} T}}{1+L_{0} T+r C_{0} T+r L_{1} T^{2}}\right\} .
$$

Consequently, there exists constants $\rho, T_{0}>0$, depending on the data $r_{0}, R, C_{0}, H_{0}, L_{0}, L_{1}$, such that, for every $T \in\left(0, T_{0}\right)$, the attainable set $\mathcal{A}(T)$ satisfies an interior sphere condition of radius $\rho_{T}=\rho T$. Knowing that $\rho_{T}$ 
is linear in $T$ - at least for $T \in\left(0, T_{0}\right)$ - could be useful to further investigate the structure of attainable sets, as shown in [6] where such a property is used to study the evolution of perimeters for attainable sets.

Example 3.7. Let us discuss some applications of the above results when our system is affine in the control variables, i.e.

$$
f(x, u)=M(x) u+g(x) \quad(x, u) \in \mathbb{R}^{n} \times U
$$

where $M(x)=\left\{a_{i j}(x)\right\}_{i, j=1}^{n}$ is an $n \times n$ matrix, $g(x)=\left(g_{1}(x), \ldots, g_{n}(x)\right)$ an $n$-dimensional vector, and $U \subset \mathbb{R}^{n}$ a compact convex set. We will show that, if

(i) $U$ has the interior $r$-sphere property ;

(ii) $\quad a_{i j}, g_{i} \in C^{1,1}\left(\mathbb{R}^{n}\right)$ for every $i, j=1, \ldots, n$;

(iii) $M(0)$ is nonsingular ;

then all the assumptions of Theorem 3.5 are satisfied taking $\mathcal{O}$ equal to a suitable open neighbourhood of 0. Indeed, conditions (6) and 7) are immediate to check. So, let us prove that $M(x) U+g(x)$ has the interior sphere property. For this, we may as well suppose $g \equiv 0$ since the interior sphere property is invariant under translations. Now, on account of (iii), there exists $R>0$ such that $\operatorname{det} M(x) \neq 0$ for every $x \in R \bar{B}$. Then, $\partial(M(x) U)=M(x)(\partial U)$ for every $x \in R \bar{B}$. Hereafter, we will only consider points $x \in R \bar{B}$.

Let $v_{0} \in \partial(M(x) U)$ and $u_{0} \in \partial U$ be such that $v_{0}=M(x) u_{0}$. Set $\bar{u}_{0}=u_{0}-r \nabla b_{U}\left(u_{0}\right)$, and observe that

$$
u_{0} \in \bar{u}_{0}+r \bar{B} \subset U
$$

in view of (i). It is easy to realize that $M(x)\left(\bar{u}_{0}+r \bar{B}\right)$ is the ellipsoid

$$
\mathcal{E}_{\Lambda(x)}\left(\bar{v}_{0}, r\right):=\left\{v \in \mathbb{R}^{n}:\left\langle\Lambda(x)\left(v-\bar{v}_{0}\right), v-\bar{v}_{0}\right\rangle \leq r^{2}\right\}
$$

where $\bar{v}_{0}=M(x) \bar{u}_{0}, M^{-1}(x)$ denotes the inverse of $M(x)$, and $\Lambda(x)=M^{-1}(x)^{\star} M^{-1}(x)$. Thus,

$$
v_{0} \in \mathcal{E}_{\Lambda(x)}\left(\bar{v}_{0}, r\right) \subset M(x) U
$$

Since, as recalled in Example 2.2, $\mathcal{E}_{\Lambda(x)}(r)$ has the interior sphere property, so does $M(x) U$.

Finally, assumption (19) is satisfied on account of Proposition 3.9 below. Indeed, the nondegeneracy of $M$ on $R \bar{B}$ implies that $f$ satisfies (34).

Let us now turn to the analysis of general attainable sets $\mathcal{A}(\mathcal{K} ; T)$. We note, first, that Theorem 3.5 can be immediately generalized to the set $\mathcal{A}(\{x\} ; T)$ for any point $x \in \mathbb{R}^{n}$. Moreover, for any closed set $\mathcal{K} \subset \mathbb{R}^{n}$,

$$
\mathcal{A}(\mathcal{K} ; T)=\bigcup_{x \in \mathcal{K}} \mathcal{A}(\{x\} ; T)
$$

Since any union of sets satisfying an interior sphere condition radius $\rho$ satisfies an interior sphere condition of the same radius, the general result below easily follows recalling Remark 3.6.

Theorem 3.8. Assume $(\mathrm{A})$, and let $\mathcal{K} \subset \mathcal{O}$ be a closed set. Suppose that

$$
|f(x, u)| \leq H \quad \forall(x, u) \in \mathcal{K} \times U
$$

for some constant $H>0$. If $x \rightsquigarrow \partial F(x)$ is a Lipschitz boundary map, then numbers $\rho, T_{0}>0$ exist such that, for all $T \in\left(0, T_{0}\right), \mathcal{A}(\mathcal{K} ; T)$ satisfies an interior sphere condition of radius $\rho T$. 


\subsection{Lipschitz boundary maps}

In this section we will discuss the notion of Lipschitz boundary map, introduced in Definition 3.4. To begin with, we observe that a similar property that could be derived just from (A0), (A1) and (A3), is that $x \rightsquigarrow \partial F(x)$ is a "Hölder boundary map" of exponent $1 / 2$, that is

$$
\left|\nabla b_{F(y)}(v)-\nabla b_{F(x)}(v)\right| \leq C_{0}|x-y|^{1 / 2}
$$

for all $x \in \mathcal{O}$, all $y \in \bar{B}\left(x, r_{0}\right) \cap \mathcal{O}$, and all $v \in \partial F(x)$. However, we will omit further details since we are interested in the above estimate with exponent 1 . We propose, instead, two different sufficient conditions to guarantee that (19) is satisfied. Our first condition is the following.

Proposition 3.9. Assume $(\mathrm{A})$ and suppose that, for some constant $C_{2} \geq 0$ and every pair $(x, u) \in \mathcal{O} \times U$ satisfying $f(x, u) \in \partial F(x)+\frac{r}{2} B$,

$$
\left|\left[D_{x} f\left(x, u^{\prime}\right)-D_{x} f(x, u)\right]^{\star} \nabla b_{F(x)}(f(x, u))\right| \leq C_{2}\left|f\left(x, u^{\prime}\right)-f(x, u)\right| \quad \forall u^{\prime} \in U .
$$

Then, $x \rightsquigarrow \partial F(x)$ is a Lipschitz boundary map.

The proof of Proposition 3.9 requires the following lemma.

Lemma 3.10. Assume $(\mathrm{A})$ and $(34)$, and define $r_{1}:=\min \left\{\frac{r}{4 L_{0}}, \frac{1}{2 C_{2}}\right\}$. Then, for all $x \in \mathcal{O}$, all $y \in B\left(x, r_{1}\right) \cap \mathcal{O}$, and all $u \in U$ satisfying $f(x, u) \in \partial F(x)$,

$$
\begin{gathered}
\left|b_{F(y)}(f(y, u))-b_{F(y)}(f(x, u))-\left\langle\nabla b_{F(y)}(f(x, u)), f(y, u)-f(x, u)\right\rangle\right| \leq \frac{2 L_{0}^{2}}{r}|x-y|^{2} \\
\left|b_{F(y)}(f(y, u))\right| \leq C_{3}|x-y|^{2}
\end{gathered}
$$

for some constant $C_{3} \geq 0$.

Proof. Let $x \in \mathcal{O}, v:=f(x, u) \in \partial F(x)$, and recall that, in view of Proposition 3.1(a), $b_{F(y)} \in C^{1,1}\left(\partial F(y)+\frac{r}{2} B\right)$ for every $y \in \mathcal{O}$. Let us also observe that, on account of $(9), f(x, u) \in \partial F(y)+\frac{r}{4} B$ and so $f(y, u) \in \partial F(y)+\frac{r}{2} B$ for every $y \in B\left(x, r_{1}\right) \cap \mathcal{O}$. Hence, owing to (10),

$$
\left|b_{F(y)}(f(y, u))-b_{F(y)}(v)-\left\langle\nabla b_{F(y)}(v), f(y, u)-v\right\rangle\right| \leq \frac{2}{r}|f(y, u)-v|^{2} .
$$

Since $|f(y, u)-v| \leq L_{0}|x-y|$, estimate (35) follows.

In order to prove (36), let $t \geq 0$ be such that $f(y, u)+t \nabla b_{F(x)}(v) \in F(y)$. Then, for some $u_{t} \in U$, $f\left(y, u_{t}\right)=f(y, u)+t \nabla b_{F(x)}(v)$. Therefore, invoking the convexity of $F(y)$ and $(34)$,

$$
\begin{aligned}
t= & \left\langle\nabla b_{F(x)}(v), f\left(y, u_{t}\right)-f(y, u)\right\rangle \\
\leq & \left\langle\nabla b_{F(x)}(v), f\left(x, u_{t}\right)-f(x, u)\right\rangle+\left\langle\nabla b_{F(x)}(v),\left[D_{x} f\left(x, u_{t}\right)-D_{x} f(x, u)\right](y-x)\right\rangle \\
& +L_{1}|x-y|^{2} \\
\leq & C_{2}\left|f\left(x, u_{t}\right)-f(x, u)\right||x-y|+L_{1}|x-y|^{2} .
\end{aligned}
$$

On the other hand,

$$
\begin{aligned}
\left|f\left(x, u_{t}\right)-f(x, u)\right| & \leq\left|f\left(x, u_{t}\right)-f\left(y, u_{t}\right)\right|+\left|f\left(y, u_{t}\right)-f(y, u)\right|+|f(y, u)-f(x, u)| \\
& \leq 2 L_{0}|x-y|+t .
\end{aligned}
$$


So, owing to our choice of $r_{1}$,

$$
t \leq\left(2 L_{0} C_{2}+L_{1}\right)|x-y|^{2}+\frac{t}{2}
$$

and (36) follows, by a maximality argument, with $C_{3}=2\left(2 L_{0} C_{2}+L_{1}\right)$.

Proof of Proposition 3.9. Define $r_{1}$ as in Lemma 3.10, and let $x \in \mathcal{O}, y \in B\left(x, r_{1}\right) \cap \mathcal{O}$, and $v=f(x, u) \in \partial F(x)$. In view of $(11), v-r \nabla b_{F(x)}(v)+r \nabla b_{F(y)}(v) \in F(x)$. So,

$$
v-r \nabla b_{F(x)}(v)+r \nabla b_{F(y)}(v)=f\left(x, u_{y}\right)
$$

for some $u_{y} \in U$. Consider the projection $v_{y}=v-b_{F(y)}(v) \nabla b_{F(y)}(v)$ of $v$ onto $\partial F(y)$. Invoking convexity and the fact that $\nabla b_{F(y)}\left(v_{y}\right)=\nabla b_{F(y)}(v)$, we obtain

$$
\begin{aligned}
0 & \geq\left\langle\nabla b_{F(y)}\left(v_{y}\right), f\left(y, u_{y}\right)-v_{y}\right\rangle \\
& =\left\langle\nabla b_{F(y)}(v), f\left(x, u_{y}\right)-v_{y}\right\rangle+\left\langle\nabla b_{F(y)}(v), f\left(y, u_{y}\right)-f\left(x, u_{y}\right)\right\rangle \\
& =-r\left\langle\nabla b_{F(y)}(v), \nabla b_{F(x)}(v)\right\rangle+r+b_{F(y)}(v)+\left\langle\nabla b_{F(y)}(v), f\left(y, u_{y}\right)-f\left(x, u_{y}\right)\right\rangle
\end{aligned}
$$

Therefore, owing to Lemma 3.10,

$$
\begin{aligned}
& r\left\langle\nabla b_{F(y)}(v), \nabla b_{F(x)}(v)\right\rangle \geq r+b_{F(y)}(f(y, u))+\left\langle\nabla b_{F(y)}(v), f(x, u)-f(y, u)\right\rangle \\
& \quad+\left\langle\nabla b_{F(y)}(v), f\left(y, u_{y}\right)-f\left(x, u_{y}\right)\right\rangle-\frac{2 L_{0}^{2}}{r}|x-y|^{2} \\
& \quad \geq r+\left\langle\nabla b_{F(y)}(v),\left[f\left(y, u_{y}\right)-f(y, u)\right]-\left[f\left(x, u_{y}\right)-f(x, u)\right]\right\rangle-\left(C_{3}+\frac{2 L_{0}^{2}}{r}\right)|x-y|^{2} .
\end{aligned}
$$

On the other hand, in view of (A2), (10), and (34), we have

$$
\begin{aligned}
& \left\langle\nabla b_{F(y)}(v),\left[f\left(y, u_{y}\right)-f(y, u)\right]-\left[f\left(x, u_{y}\right)-f(x, u)\right]\right\rangle \\
& \quad \geq\left\langle\nabla b_{F(y)}(f(x, u)),\left[D_{x} f\left(y, u_{y}\right)-D_{x} f(y, u)\right](y-x)\right\rangle-L_{1}|x-y|^{2} \\
& \quad \geq\left\langle\nabla b_{F(y)}(f(y, u)),\left[D_{x} f\left(y, u_{y}\right)-D_{x} f(y, u)\right](y-x)\right\rangle-\left(\frac{4 L_{0}}{r}+L_{1}\right)|x-y|^{2} \\
& \quad \geq-C_{2}\left|f\left(y, u_{y}\right)-f(y, u)\right||x-y|-\left(\frac{4 L_{0}}{r}+L_{1}\right)|x-y|^{2}
\end{aligned}
$$

Moreover,

$$
\begin{aligned}
\left|f\left(y, u_{y}\right)-f(y, u)\right| & \leq\left|f\left(y, u_{y}\right)-f\left(x, u_{y}\right)\right|+\left|f\left(x, u_{y}\right)-f(x, u)\right|+|f(x, u)-f(y, u)| \\
& \leq 2 L_{0}|x-y|+r\left|\nabla b_{F(x)}(v)-\nabla b_{F(y)}(v)\right| .
\end{aligned}
$$

Now, combine the above estimates with the binomial inequality $\alpha \beta \leq \frac{\alpha^{2}}{4}+\beta^{2}$, to conclude that

$$
\begin{aligned}
& r\left\langle\nabla b_{F(y)}(v), \nabla b_{F(x)}(v)\right\rangle \\
& \quad \geq r-r C_{2}\left|\nabla b_{F(x)}(v)-\nabla b_{F(y)}(v)\right||x-y|-\left(L_{1}+C_{3}+2 L_{0} C_{2}+\frac{4 L_{0}}{r}+\frac{2 L_{0}^{2}}{r}\right)|x-y|^{2} \\
& \quad \geq r-\frac{r}{4}\left|\nabla b_{F(x)}(v)-\nabla b_{F(y)}(v)\right|^{2}-\left(L_{1}+C_{3}+2 L_{0} C_{2}+r C_{2}^{2}+\frac{4 L_{0}}{r}+\frac{2 L_{0}^{2}}{r}\right)|x-y|^{2} .
\end{aligned}
$$


Thus, recalling that $\nabla b_{F(x)}$ and $\nabla b_{F(y)}$ are unit vectors,

$$
\begin{aligned}
\left|\nabla b_{F(x)}(v)-\nabla b_{F(y)}(v)\right|^{2} & =2\left(1-\left\langle\nabla b_{F(y)}(v), \nabla b_{F(x)}(v)\right\rangle\right) \\
& \leq \frac{1}{2}\left|\nabla b_{F(x)}(v)-\nabla b_{F(y)}(v)\right|^{2}+C_{4}|x-y|^{2},
\end{aligned}
$$

where $C_{4}=\frac{2}{r}\left(L_{1}+C_{3}+2 L_{0} C_{2}+r C_{2}^{2}+\frac{4 L_{0}}{r}+\frac{2 L_{0}^{2}}{r}\right)$. The conclusion follows, with $C_{0}=\sqrt{2 C_{4}}$.

Our second sufficient condition for $x \rightsquigarrow \partial F(x)$ to satisfy (19) is expressed in Hamiltonian form. Let

$$
H(x, p):=\max _{v \in F(x)}\langle v, p\rangle \quad x, p \in \mathbb{R}^{n}
$$

be the Hamiltonian associated with $F(x)$, and denote by $S^{n-1}$ the unit sphere in $\mathbb{R}^{n}$.

Proposition 3.11. Under assumptions $(A)$, suppose $H$ is differentiable with respect to $p$ on $\mathcal{O} \times\left(\mathbb{R}^{n} \backslash\{0\}\right)$, and $\nabla_{p} H(\cdot, p)$ is Lipschitz continuous on $\mathcal{O}$, uniformly in $p \in S^{n-1}$. Then, $x \rightsquigarrow \partial F(x)$ is a Lipschitz boundary map.

Proof. Observe that, for all $(x, p) \in \mathcal{O} \times S^{n-1}, H(x, p)=\left\langle v_{x, p}, p\right\rangle$ for some $v_{x, p} \in \partial F(x)$. Moreover, for any such vector $v_{x, p}$, we have that $p=\nabla b_{F(x)}\left(v_{x, p}\right)$ and $\nabla_{p} H(x, p)=v_{x, p}$. Therefore, for all $x \in \mathcal{O}$ and $p, q \in S^{n-1}$, inequality (10) yields

$$
|p-q| \leq \frac{2}{r}\left|\nabla_{p} H(x, p)-\nabla_{p} H(x, q)\right| .
$$

Now, fix $x \in \mathcal{O}, y \in \bar{B}\left(x, \frac{r}{2 L_{0}}\right)$, and $v \in \partial F(x)$. Let $w$ be the projection of $v$ onto $\partial F(y)$. Consider the unit vectors $p:=\nabla b_{F(x)}(v)$ and $q:=\nabla b_{F(y)}(w)=\nabla b_{F(y)}(v)$. Then, in view of (37) we have

$$
\begin{aligned}
\left|\nabla b_{F(y)}(v)-\nabla b_{F(x)}(v)\right| & \leq \frac{2}{r}\left|\nabla_{p} H(x, p)-\nabla_{p} H(x, q)\right| \\
& \leq \frac{2}{r}\left(\left|\nabla_{p} H(x, p)-\nabla_{p} H(y, q)\right|+L|x-y|\right)
\end{aligned}
$$

where $L$ denotes a Lipschitz constant for $\nabla_{p} H(\cdot, q)$. Since $\nabla_{p} H(x, p)=v$ and $\nabla_{p} H(y, q)=w,(9)$ ensures

$$
\left|\nabla_{p} H(x, p)-\nabla_{p} H(y, q)\right|=\left|b_{F(y)}(v)\right| \leq L_{0}|x-y| .
$$

The conclusion follows from the last two inequalities above.

\section{4. $C^{1,1}$ regularity}

We conclude this section with a $C^{1,1}$ regularity result for attainable sets. We recall that a subset $S$ of $\mathbb{R}^{n}$ is called $a$-regular, for some given real number $a>0$, if, for all points $x_{0}, x_{1} \in S$ and numbers $\lambda \in(0,1)$, the closed ball

$$
\left\{x \in \mathbb{R}^{n}:\left|x-\lambda x_{1}-(1-\lambda) x_{0}\right| \leq a \lambda(1-\lambda)\left|x_{1}-x_{0}\right|^{2}\right\}
$$

is contained in $S$. Clearly, any $a$-regular set is convex.

Corollary 3.12. Assume (A) and (33), where $\mathcal{K} \subset \mathcal{O}$ is a closed set. Suppose that, for some $a>0, \mathcal{K}$ and $F(x)$ are a-regular for every $x \in \mathcal{O}$. If $x \rightsquigarrow \partial F(x)$ is a Lipschitz boundary map, then there exists $T_{1}>0$ such that, for every $T \in\left(0, T_{1}\right)$, the oriented distance $b_{\mathcal{A}(\mathcal{K} ; T)}$ is of class $C^{1,1}$ on some neighborhood of $\partial \mathcal{A}(\mathcal{K} ; T)$. In particular, $\partial \mathcal{A}(\mathcal{K} ; T) \in C^{1,1}$ for all $T \in\left(0, T_{1}\right)$.

Proof. In view of Theorem 3.8 we have that $\mathcal{A}(\mathcal{K} ; T)$ satisfies a uniform interior sphere condition for every $T \in\left(0, T_{0}\right)$. Moreover, for $T$ sufficiently small, $\mathcal{A}(\mathcal{K} ; T)$ is convex as shown in [18]. Then, the conclusion follows arguing as in the proof of Proposition 3.1(a). 


\section{A counterexample}

This section completes the previous one showing that the result of Theorem 3.5 is sharp. Indeed, we will construct a control system $\dot{y}=f(y, u)$, with $f(\cdot, u)$ Lipschitz and $f(x, U)$ satisfying a uniform interior sphere condition, such that, for all sufficiently small $t>0$, the attainable set at time $t$ fails to possess such a property.

Consider the control system in $\mathbb{R}^{n}, n \geq 2$,

$$
\left\{\begin{array}{l}
\dot{y}=(1-|y|) u(s)-q_{0}, \quad u(s) \in \bar{B} \\
y(0)=0
\end{array}\right.
$$

where $q_{0}$ is a unit vector. Observe that $y \equiv 0$ is a solution to the above system corresponding to the constant control $u=q_{0}$ and, for all small $t>0$, the origin belongs to the boundary of $\mathcal{A}(t)$. Indeed, for every solution $y$ to (38) and $t>0$ small enough,

$$
\left\langle q_{0}, y(t)\right\rangle=\int_{0}^{t}\left\langle q_{0},(1-|y(s)|) u(s)\right\rangle \mathrm{d} s-t \leq \int_{0}^{t}(1-|y(s)|) \mathrm{d} s-t \leq 0 .
$$

This implies that zero is a boundary point of $\mathcal{A}(t)$.

Clearly the sets $F(x):=(1-|x|) \bar{B}-q_{0}$ satisfy an interior sphere condition of radius $1 / 2$ for all $x \in B\left(\frac{1}{2}\right)$.

Let $t>0$ be such that $t e^{t}<\frac{1}{4}$ and every solution $y(\cdot)$ to (38) verifies $y([0, t]) \subset B\left(\frac{1}{2}\right)$.

Claim 1. The reachable set $\mathcal{A}(t)$ of (38) is convex and compact.

The compactness of $\mathcal{A}(t)$ was recalled in Section 3. As for its convexity, we note that the set of all trajectories of (38) is convex. Indeed, since map $x \mapsto 1-|x|$ is concave, for any pair of solutions $y_{0}, y_{1}$ to (38) and any $\lambda \in[0,1]$, we have

$$
\begin{array}{r}
\left(\lambda y_{1}+(1-\lambda) y_{0}\right)^{\prime}(t) \in\left(\lambda\left(1-\left|y_{1}(t)\right|\right)+(1-\lambda)\left(1-\left|y_{0}(t)\right|\right)\right) B-q_{0} \\
\subset\left(1-\left|\lambda y_{1}(t)+(1-\lambda) y_{0}(t)\right|\right) B-q_{0} .
\end{array}
$$

Claim 2. If $y$ is a solution to $(38)$ and $y(t)=0$, then $y \equiv 0$ in $[0, t]$.

Indeed, by the nonsmooth Maximum Principle (see, for instance, [13]), there exists a nonvanishing absolutely continuous function $p(s)$ satisfying, for a.e. $s \in[0, t]$,

$$
\dot{y}(s)=(1-|y(s)|) \frac{p(s)}{|p(s)|}-q_{0}
$$

Consider $\tilde{y}=\frac{1}{2} y$. Since $\tilde{y}(t)=0 \in \partial \mathcal{A}(t)$, again by the Maximum Principle, there exists a control $u$, with $|u(s)|=1$, such that, for a.e. $s \in[0, t]$,

$$
\tilde{y}^{\prime}(s)=\left(1-\frac{1}{2}|y(s)|\right) u(s)-q_{0}=\frac{1}{2}(1-|y(s)|) \frac{p(s)}{|p(s)|}-\frac{1}{2} q_{0} .
$$

So,

whence, since $|u(s)|=1$,

$$
\frac{1}{2}(1-|y(s)|) \frac{p(s)}{|p(s)|}+\frac{1}{2} q_{0}=\left(1-\frac{1}{2}|y(s)|\right) u(s)
$$

$$
1-\frac{1}{2}|y(s)|=\left|\frac{1}{2}(1-|y(s)|) \frac{p(s)}{|p(s)|}+\frac{1}{2} q_{0}\right| \leq \frac{1}{2}(1-|y(s)|)+\frac{1}{2}=1-\frac{1}{2}|y(s)| .
$$

This yields $\frac{p(s)}{|p(s)|}=q_{0}$ for a.e. $s \in[0, t]$. But $\frac{p(s)}{|p(s)|}$ is the control corresponding to $y$ and $q_{0}$ is the control corresponding to the zero trajectory. So $y=0$ on $[0, t]$. 
Claim 3. $N_{\mathcal{A}(t)}(0)$ contains exactly one unit vector, namely $q_{0}$.

Indeed, let $p_{0} \in N_{\mathcal{A}(t)}(0)$ be a unit vector and $y(\cdot)$ be the solution of (38) with $u \equiv p_{0}$. Note that $y(s) \in \mathcal{A}(t)$, hence $\left\langle p_{0}, y(s)\right\rangle \leq 0$, for every $s \in[0, t]$. Moreover, $\left\langle p_{0}, y(0)\right\rangle=0$. Therefore,

$$
0 \geq\left.\frac{\mathrm{d}}{\mathrm{d} s}\left\langle p_{0}, y(s)\right\rangle\right|_{s=0}=1-\left\langle p_{0}, q_{0}\right\rangle
$$

and so $p_{0}=q_{0}$ since $p_{0}$ and $q_{0}$ are unit vectors.

We will now complete the analysis of system $(38)$ showing that $\mathcal{A}(t)$ fails to satisfy an interior sphere condition. This will follow from Proposition 2.3 provided we can construct a sequence $\left\{y_{i}\right\}$ of boundary trajectories satisfying $0 \neq y_{i}(t) \rightarrow 0$ as $i \rightarrow \infty$, and

$$
\lim _{i \rightarrow \infty} \frac{\left\langle q_{0},-y_{i}(t)\right\rangle}{\left|y_{i}(t)\right|^{2}}=+\infty .
$$

In fact, we will prove that (39) is true for any sequence of boundary trajectories such that $0 \neq y_{i}(t) \rightarrow 0$ as $i \rightarrow \infty$. Indeed, let $\left\{y_{i}\right\}$ be any such sequence (here, the fact that $n \geq 2$ is used), let $u_{i}(\cdot)$ be the corresponding controls, and recall that $\left|u_{i}(s)\right|=1$ for a.e. $s \in[0, t]$.

Claim 4. $\left\{u_{i}\right\}$ converges to the constant vector $q_{0}$ uniformly in $[0, t]$.

Indeed, by the nonsmooth Maximum Principle there exist adjoint states $p_{i}(\cdot)$ corresponding to $y_{i}$ such that $\left|p_{i}(t)\right|=1, p_{i}(t) \in N_{\mathcal{A}(t)}\left(y_{i}(t)\right)$ and $p_{i}$ are Lipschitz with the same constant. From Claim 3 we deduce that $p_{i}(t) \rightarrow q_{0}$. By the Maximum Principle,

$$
u_{i}(s)=\frac{p_{i}(s)}{\left|p_{i}(s)\right|} \quad \text { for a.e. } \quad s \in[0, t] .
$$

From the Lipschitz continuity of $p_{i}$ we deduce that the above identity holds for all $s \in[0, t]$ and that $u_{i}$ are Lipschitz continuous with the same Lipschitz constant. Since $u_{i}(t) \rightarrow q_{0}$ we deduce that the set $\left\{u_{i}\right\}_{i>1}$ is precompact in $C([0, t])$. Let $\bar{u}$ be a cluster point of this set and $\bar{y}$ be the corresponding trajectory. Then, $\bar{y}(t)=0$. Therefore, by Claim $2, \bar{y} \equiv 0$. Consequently, $\bar{u} \equiv q_{0}$. This implies that $u_{i} \rightarrow q_{0}$ uniformly on $[0, t]$.

Claim 5. The map $s \mapsto\left|y_{i}(s)\right|$ is nondecreasing.

Indeed, from [16] it follows that $p_{i}(s) \in N_{\mathcal{A}(s)}\left(y_{i}(s)\right)$ for a.e. $s \in[0, t]$. Therefore,

$$
\left\langle\frac{p_{i}(s)}{\left|p_{i}(s)\right|}, y_{i}(s)\right\rangle \geq 0
$$

by definition. Similarly, $\left\langle q_{0}, y_{i}(s)\right\rangle \leq 0$. Thus, from Claim 1 and Claim 3, we obtain

$$
\left\langle\dot{y}_{i}(s), y_{i}(s)\right\rangle=\left\langle\left(1-\left|y_{i}(s)\right|\right) \frac{p_{i}(s)}{\left|p_{i}(s)\right|}-q_{0}, y_{i}(s)\right\rangle \geq 0,
$$

which in turn proves our claim.

Now, let $\beta_{i}(s) \in[-1,1], v_{i}(s) \in\left\{q_{0}\right\}^{\perp}$ and $\alpha_{i}(s) \geq 0$ be such that

$$
\left|v_{i}(s)\right|=1, \quad \alpha_{i}(s)^{2}+\beta_{i}(s)^{2}=1 \quad \text { and } \quad u_{i}(s)=\alpha_{i}(s) v_{i}(s)+\beta_{i}(s) q_{0} \quad(s \in[0, T]) .
$$

By Claim $4, \beta_{i}(\cdot) \rightarrow 1$ uniformly on $[0, t]$. Thus, $\alpha_{i} \rightarrow 0$ uniformly, implying that

$$
\lim _{i \rightarrow \infty} \frac{\int_{0}^{t}\left|y_{i}(s)\right| \alpha_{i}(s) \mathrm{d} s}{\int_{0}^{t}\left|y_{i}(s)\right| \mathrm{d} s}=0 .
$$


By Claim 5,

$$
\left|y_{i}(s)\right| \geq\left|\int_{0}^{s}\left(u_{i}(\tau)-q_{0}\right) \mathrm{d} \tau\right|-\int_{0}^{s}\left|y_{i}(\tau)\right| \mathrm{d} \tau \geq\left|\int_{0}^{s}\left(u_{i}(\tau)-q_{0}\right) \mathrm{d} \tau\right|-s\left|y_{i}(s)\right| .
$$

We complete $\left\{q_{0}\right\}$ to an orthogonal basis of $\mathbb{R}^{n}$. In this basis we write $v_{i}(s)=\left(v_{i}^{1}(s), \ldots, v_{i}^{n-1}(s), 0\right)$. Then, recalling that $s<1$, we have

$$
\left|y_{i}(s)\right| \geq \frac{1}{2}\left|\int_{0}^{s}\left(u_{i}(\tau)-q_{0}\right) \mathrm{d} \tau\right| \geq \frac{1}{2}\left|\int_{0}^{s} \alpha_{i}(\tau) v_{i}^{j}(\tau) \mathrm{d} \tau\right|
$$

for all $1 \leq j \leq n-1$. Consequently,

$$
\int_{0}^{t}\left|y_{i}(s)\right| \mathrm{d} s \geq \frac{1}{2} \int_{0}^{t}\left|\int_{0}^{s} \alpha_{i}(\tau) v_{i}^{j}(\tau) \mathrm{d} \tau\right| \mathrm{d} s .
$$

In order to prove (39), observe that

$$
\left\langle q_{0},-y_{i}(t)\right\rangle=\int_{0}^{t}\left(1-\beta_{i}(s)\right) \mathrm{d} s+\int_{0}^{t}\left|y_{i}(s)\right| \beta_{i}(s) \mathrm{d} s
$$

and

$$
\left|y_{i}(t)\right|^{2}=\left\langle q_{0}, y_{i}(t)\right\rangle^{2}+\left|\int_{0}^{t}\left(1-\left|y_{i}(s)\right|\right) \alpha_{i}(s) v_{i}(s) \mathrm{d} s\right|^{2} .
$$

Hence, to obtain (39), it is enough to show that

$$
\lim _{i \rightarrow \infty} \frac{\left|\int_{0}^{t}\left(1-\left|y_{i}(s)\right|\right) \alpha_{i}(s) v_{i}(s) \mathrm{d} s\right|^{2}}{\int_{0}^{t}\left(1-\beta_{i}(s)\right) \mathrm{d} s+\int_{0}^{t}\left|y_{i}(s)\right| \beta_{i}(s) \mathrm{d} s}=0 .
$$

Since, for $i$ large enough,

$$
\int_{0}^{t}\left|y_{i}(s)\right| \beta_{i}(s) \mathrm{d} s \geq \inf _{s \in[0, t]} \beta_{i}(s) \int_{0}^{t}\left|y_{i}(s)\right| \mathrm{d} s,
$$

(40) implies that, in order to prove (43), it suffices to show that

$$
\lim _{i \rightarrow \infty} \frac{\left|\int_{0}^{t} \alpha_{i}(s) v_{i}(s) \mathrm{d} s\right|^{2}}{\int_{0}^{t}\left|y_{i}(s)\right| \mathrm{d} s}=0 .
$$

But

$$
\left|\int_{0}^{t} \alpha_{i}(s) v_{i}(s) \mathrm{d} s\right|^{2}=\sum_{j=1}^{n-1}\left|\int_{0}^{t} \alpha_{i}(s) v_{i}^{j}(s) \mathrm{d} s\right|^{2} .
$$

Therefore, by (42), it is enough to show that

$$
\lim _{i \rightarrow \infty} \frac{\left|\int_{0}^{t} \alpha_{i}(s) v_{i}^{j}(s) \mathrm{d} s\right|^{2}}{\int_{0}^{t}\left|\int_{0}^{s} \alpha_{i}(\tau) v_{i}^{j}(\tau) \mathrm{d} \tau\right| \mathrm{d} s}=0
$$


where we have set $\frac{0}{0}=0$. Notice that

$$
\begin{aligned}
\left(\int_{0}^{t} \alpha_{i}(s) v_{i}^{j}(s) \mathrm{d} s\right)^{2} & =\int_{0}^{t} \frac{\mathrm{d}}{\mathrm{d} s}\left(\int_{0}^{s} \alpha_{i}(\tau) v_{i}^{j}(\tau) \mathrm{d} \tau\right)^{2} \mathrm{~d} s \\
& =2 \int_{0}^{t} \alpha_{i}(s) v_{i}^{j}(s) \int_{0}^{s} \alpha_{i}(\tau) v_{i}^{j}(\tau) \mathrm{d} \tau \mathrm{d} s \leq 2 \sup _{s \in[0, t]} \alpha_{i}(s) \int_{0}^{t}\left|\int_{0}^{s} \alpha_{i}(\tau) v_{i}^{j}(\tau) \mathrm{d} \tau\right| \mathrm{d} s
\end{aligned}
$$

Since $\left\{\alpha_{i}\right\}$ converges uniformly to zero, the last inequality yields (45).

\section{Application to time optimal control}

The subject of this last section is strictly related to the analysis of Section 3 . The control system we will be concerned with is again (8), still subject to assumptions (A). There will be, however, an important difference in perspective: in Section 3 we studied the attainable set $\mathcal{A}(\mathcal{K} ; T)$ from a given closed set $\mathcal{K} \subset \mathcal{O}$; here, we will rather consider the controllable set to $\mathcal{K}$ in time $T$, that is, the set of all points $x \in \mathbb{R}^{n}$ such that $y(t ; x, u) \in \mathcal{K}$ for some $t \in[0, T]$ and some control $u$.

More specifically, for any control $u:[0, \infty) \rightarrow U$, let us consider the transfer time to $\mathcal{K}$, i.e.,

$$
\tau_{\mathcal{K}}(x, u)=\inf \{t \geq 0: y(t ; x, u) \in \mathcal{K}\}
$$

where $\tau_{\mathcal{K}}(x, u)=\infty$ if there exists no control steering $y(t ; x, u)$ to $\mathcal{K}$ in finite time. Then, the controllable set to $\mathcal{K}$, hereafter denoted by $\mathcal{R}(\mathcal{K})$, is defined as the set of all points $x \in \mathbb{R}^{n}$ such that $\tau_{\mathcal{K}}(x, u)<\infty$ for some control $u$.

The time optimal control problem for system (8), with target $\mathcal{K}$, consists in minimizing the transfer time to $\mathcal{K}$ over all admissible controls. This classical problem, investigated from several points of view, is the object of an immense literature, see, e.g., the monographs $[3,9,11,14]$. A well-known result guarantees that time optimal controls - that is, controls minimizing $\tau_{\mathcal{K}}(x, \cdot)$ - do exist for any $x \in \mathcal{R}(\mathcal{K})$.

The value function of the above problem, namely

$$
V_{\mathcal{K}}(x)=\inf _{u(\cdot)} \tau_{\mathcal{K}}(x, u) \quad \forall x \in \mathcal{R}(\mathcal{K}),
$$

is the so-called Minimum Time function. Its level set

$$
\mathcal{R}(\mathcal{K} ; t):=\left\{x \in \mathbb{R}^{n}: V_{\mathcal{K}}(x) \leq t\right\} \quad(t \geq 0)
$$

is the so-called controllable set to $\mathcal{K}$ in time $\leq t$. Moreover, $\mathcal{R}(\mathcal{K} ; t)$ is closed and $\partial \mathcal{R}(\mathcal{K} ; t) \subset\left\{x \in \mathbb{R}^{n}:\right.$ $\left.V_{\mathcal{K}}(x)=t\right\}$ whenever $V_{\mathcal{K}}$ is upper semicontinuous.

Guaranteeing sufficient regularity properties of $V_{\mathcal{K}}$ is one of the basic issues of modern Dynamic Programming. While mild controllability assumptions on $\partial \mathcal{K}$ ensure that the Minimum Time function is continuous in $\mathcal{R}(\mathcal{K})$ (see, e.g., [3]), the situation is different for Lipschitz continuity. The first Lipschitz continuity result for $V_{\mathcal{K}}$ was obtained by Petrov in [17], for the case of $\mathcal{K}=\{0\}$, under the so-called positive-basis condition. Such a result was then extended to more general target sets in $[4,8,21,22]$. For any closed target $\mathcal{K}$, it turns out that $V_{\mathcal{K}}$ is locally Lipschitz in $\mathcal{R}(\mathcal{K})$ (and $\mathcal{R}(\mathcal{K})$ is open), provided that

$$
\exists \mu>0 \quad \text { such that } \min _{u \in U}\langle f(x, u), p\rangle<-\mu|p|, \quad \forall x \in \partial \mathcal{K}, \forall p \in N_{\mathcal{K}}(x)
$$

where $N_{\mathcal{K}}(x)$ denotes the normal cone to $\mathcal{K}$ at $x$. Moreover, condition (46) is satisfied if and only if $V_{\mathcal{K}}(x) \leq$ $C d(x, \mathcal{K})$ holds, for some constant $C \geq 0$, in a neighbourhood of $\mathcal{K}$.

Stronger regularity properties of the Minimum Time function are harder to recover, since $V_{\mathcal{K}}$ is known to be, in general, a nonsmooth function even for very smooth data. The maximal kind of regularity we can expect for 
$V_{\mathcal{K}}$ is local semiconcavity in $\mathcal{R}(\mathcal{K}) \backslash \mathcal{K}$ (see Sect. 2 for the definition of semiconcave functions). Since semiconcave functions are locally Lipschitz continuous, condition (46) is necessary, in some sense, for $V_{\mathcal{K}}$ to be semiconcave. The result below, ensuring that such a condition is also sufficient when $\mathcal{K}$ satisfies a uniform interior sphere condition, follows from [8].

Proposition 5.1. Assume $(\mathrm{A}),(33)$ and (46). If $\mathcal{K}$ satisfies a uniform interior sphere condition, then $V_{\mathcal{K}}$ is semiconcave in $\mathcal{R}(\mathcal{K}) \backslash \mathcal{K}$.

Actually, in [8], condition (46) is imposed for every proximal normal to $\mathcal{K}$, rather than for every $\nu \in N_{\mathcal{K}}(x)$ as we do here. However, these two formulations of the controllability property are equivalent in our context, see the Appendix.

The main result of this section is the following alternative criterion for the semiconcavity of $V_{\mathcal{K}}$. Here, we assume that the velocity sets $f(x, U)$, instead of $\mathcal{K}$, satisfy a uniform interior sphere condition. Notice that $V_{\mathcal{K}}$ may fail to be semiconcave when neither $\mathcal{K}$ nor $f(x, U)$ satisfy an interior sphere condition, as shown in Example 5.4 below.

Theorem 5.2. Assume (A), (33) and (46). If $x \rightsquigarrow \partial F(x)$ is a Lipschitz boundary map, then $V_{\mathcal{K}}$ is locally semiconcave in $\mathcal{R}(\mathcal{K}) \backslash \mathcal{K}$.

The proof of Theorem 5.2 relies on the following lemma.

Lemma 5.3. Assume (A), (33) and (46). Then, for any $t>0$ sufficiently small,

$$
\min _{u \in U}\langle f(x, u), p\rangle<-\frac{\mu}{2}|p|, \quad \forall x \in \partial \mathcal{R}(\mathcal{K} ; t), \forall p \in N_{\mathcal{R}(\mathcal{K} ; t)}(x) .
$$

Proof. Let $\bar{x} \in \partial \mathcal{R}(\mathcal{K} ; t)$ be fixed. Since $V_{\mathcal{K}}$ is continuous, $V_{\mathcal{K}}(\bar{x})=t$. Let $\bar{u}(\cdot)$ be time optimal at $\bar{x}$, i.e., $y(t ; \bar{x}, \bar{u}) \in \mathcal{K}$. Then, the $\operatorname{arc} x(s):=y(t-s ; \bar{x}, \bar{u})$ satisfies

$$
\left\{\begin{array}{l}
\dot{x}(s)=-f(x(s), u(s)) \quad s \in(0, t) \text { a.e. } \\
x(0) \in \mathcal{K} \\
x(t)=\bar{x}
\end{array}\right.
$$

where $u(s)=\bar{u}(t-s)$. Moreover, by assumptions (A) and (33), we have

$$
|x(s)-x(0)| \leq t H \mathrm{e}^{t L_{0}} \quad \forall s \in[0, t] .
$$

Now, denote by $X(\cdot)$ the matrix solution of

$$
\left\{\begin{array}{l}
X^{\prime}(s)=-D_{x} f(x(s), u(s)) X(s) \quad(0<s<t) \\
X(0)=I
\end{array}\right.
$$

Again, observe that

$$
\|X(s)-I\| \leq t L_{0} \mathrm{e}^{t L_{0}} \quad \forall s \in[0, t] .
$$

By well-known properties of the variational equation, we have that

$$
w \in T_{\mathcal{K}}(x(0)) \quad \Longrightarrow \quad X(t) w \in T_{\mathcal{R}(\mathcal{K} ; t)}(x(t)) .
$$

Therefore, $\langle p, X(t) w\rangle \leq 0$ for all $w \in T_{\mathcal{K}}(x(0))$ and $p \in N_{\mathcal{R}(\mathcal{K} ; t)}(\bar{x})$. Hereby, $X^{*}(t) p \in N_{\mathcal{K}}(x(0))$ for all $p \in N_{\mathcal{R}(\mathcal{K} ; t)}(\bar{x})$.

Let $\bar{p}$ be a fixed unit vector of $N_{\mathcal{R}(\mathcal{K} ; t)}(\bar{x})$. Then, in view of $(46)$,

$$
\left\langle f(x(0), \bar{u}), X^{*}(t) \bar{p}\right\rangle<-\mu\left|X^{*}(t) \bar{p}\right|
$$


for some $\bar{u} \in U$. We claim that

$$
\langle f(\bar{x}, \bar{u}), \bar{p}\rangle<-\frac{\mu}{2} \quad \forall t \in\left(0, \frac{\mu}{2 e L_{0}(2 M+\mu)}\right) .
$$

Indeed, owing to (47), (48) and (49),

$$
\begin{aligned}
\langle f(\bar{x}, \bar{u}), \bar{p}\rangle & \leq\langle f(x(0), \bar{u}), \bar{p}\rangle+t L_{0} H \mathrm{e}^{t L_{0}} \\
& \leq-\mu\left|X^{*}(t) \bar{p}\right|+2 t L_{0} H \mathrm{e}^{t L_{0}} \leq t L_{0} \mathrm{e}^{t L_{0}}(2 H+\mu)-\mu .
\end{aligned}
$$

Our claim follows noting that $t L_{0} \mathrm{e}^{t L_{0}}(2 H+\mu) \leq \frac{\mu}{2}$ when $t$ is chosen as in (50).

Proof of Theorem 5.2. Let us observe, first, that $\partial \mathcal{R}(\mathcal{K} ; t) \subset \partial \mathcal{A}(\mathcal{K} ; t)$, where $\mathcal{A}(\mathcal{K} ; t)$ now denotes the attainable set for the control system associated with $-f$. Indeed, let $\bar{x} \in \partial \mathcal{R}(\mathcal{K} ; t)$. Arguing as in the proof of the previous lemma we conclude that $\bar{x} \in \mathcal{A}(\mathcal{K} ; t)$. Moreover, $\bar{x}$ must be on the boundary of $\mathcal{A}(\mathcal{K} ; t)$ for otherwise there would be a ball $B_{\rho}(\bar{x}) \subset \mathcal{A}(\mathcal{K} ; t)$. Then, the same standard argument as above, based on time inversion, would show that $V_{\mathcal{K}}(x) \leq t$ for all the points $x$ of such a ball, contradicting $\bar{x} \in \partial \mathcal{R}(\mathcal{K} ; t)$.

Therefore, in view of Theorem 3.8, we conclude that $\mathcal{R}(\mathcal{K} ; t)$ satisfies a uniform interior sphere condition for $t$ sufficiently small, say $0<t<t_{0}$. Moreover, invoking Lemma 5.3 , we have that $\mathcal{R}(\mathcal{K} ; t)$ satisfies (46), with $\mu$ replaced by $\mu / 2$, for all $t$ of a possibly smaller interval $\left(0, t_{1}\right)$. Therefore, by Proposition 5.1 , the Minimum Time function with target $\mathcal{R}(\mathcal{K} ; t), V_{\mathcal{R}(\mathcal{K} ; t)}$, is semiconcave in $\mathcal{R}(\mathcal{K}) \backslash \mathcal{R}(\mathcal{K} ; t)$ for every $t \in\left(0, t_{1}\right)$.

Next, we claim that, for any $t \in\left(0, t_{1}\right)$,

$$
V_{\mathcal{K}}(x)=V_{\mathcal{R}(\mathcal{K} ; t)}(x)+t \quad \forall x \in \mathcal{R}(\mathcal{K}) \backslash \mathcal{R}(\mathcal{K} ; t) .
$$

Indeed, the optimality principle implies that, for any $x \in \mathcal{R}(\mathcal{K}) \backslash \mathcal{R}(\mathcal{K} ; t)$ and any control $u(\cdot)$,

$$
V_{\mathcal{K}}(x) \leq \tau_{\mathcal{R}(\mathcal{K} ; t)}(x, u)+V_{\mathcal{K}}\left(y\left(\tau_{\mathcal{R}(\mathcal{K} ; t)}(x, u) ; x, u\right)\right) \leq \tau_{\mathcal{R}(\mathcal{K} ; t)}(x, u)+t,
$$

whence $V_{\mathcal{K}}(x) \leq V_{\mathcal{R}(\mathcal{K} ; t)}(x)+t$. For the converse inequality, let $u_{*}(\cdot)$ be time-optimal at $x$ and denote by $y_{*}(\cdot)$ the corresponding trajectory. Since $y_{*}\left(\tau_{\mathcal{R}(\mathcal{K} ; t)}\left(x, u_{*}\right)\right) \in \partial \mathcal{R}(\mathcal{K} ; t)$ and $V_{\mathcal{K}}$ is continuous, $V_{\mathcal{K}}\left(y_{*}\left(\tau_{\mathcal{R}(\mathcal{K} ; t)}\left(x, u_{*}\right)\right)\right)=t$. Thus, again the optimality principle yields

$$
V_{\mathcal{K}}(x)=\tau_{\mathcal{R}(\mathcal{K} ; t)}\left(x, u_{*}\right)+t \geq V_{\mathcal{R}(\mathcal{K} ; t)}(x)+t,
$$

which proves our claim.

Now, in view of $(51), V_{\mathcal{K}}$ is semiconcave in $\mathcal{R}(\mathcal{K}) \backslash \mathcal{R}(\mathcal{K} ; t)$ since so is $V_{\mathcal{R}(\mathcal{K} ; t)}$. Moreover, this must be true for every $t \in\left(0, t_{1}\right)$. But $\{\mathcal{R}(\mathcal{K} ; t)\}$ is an increasing family of sets, and

$$
\bigcap_{0<t<t_{1}} \mathcal{R}(\mathcal{K} ; t)=\mathcal{K}
$$

Hence, $V_{\mathcal{K}}$ is locally semiconcave in $\mathcal{R}(\mathcal{K}) \backslash \mathcal{K}$.

Example 5.4. Consider the control system $\dot{y}=u$, with $y \in \mathbb{R}^{2}$ and $u(t) \in U=[-1,1] \times[-1,1]$. Taking $\mathcal{K}=\{(0,0)\}$ as our target, we have that

$$
V_{\mathcal{K}}\left(x_{1}, x_{2}\right)=\max \left\{\left|x_{1}\right|,\left|x_{2}\right|\right\} .
$$

Since $V_{\mathcal{K}}$ is convex and not differentiable at all points $\left(x_{1}, x_{2}\right)$ with $\left|x_{1}\right|=\left|x_{2}\right|, V_{\mathcal{K}}$ cannot be semiconcave. Observe, also, that the points where $V_{\mathcal{K}}$ is not differentiable are exactly the ones where the time optimal trajectory is unique. 
Remark 5.5. For certain optimal exit time problems including the minimum time problem, Sinestrari [20] obtained a semiconcavity result under the same assumptions of Theorem 5.2, except for (19) that he replaced with the condition

$$
\left\|D_{x} f\left(x, u^{\prime}\right)-D_{x} f(x, u)\right\| \leq C\left|f\left(x, u^{\prime}\right)-f(x, u)\right| \quad \forall x \in \mathcal{O}, \forall u, u^{\prime} \in U
$$

for some constant $C \geq 0$. It is then straightforward to show that the level sets $\mathcal{R}(\mathcal{K} ; t)$ satisfy a uniform interior sphere condition for $t>0$.

On the one hand, let us note that the above condition is stronger than (34). So, as shown by Proposition 3.9, it is also stronger than requiring $x \rightsquigarrow \partial F(x)$ to be a Lipschitz boundary map.

On the other hand, we would like to point out that the perspective of the present paper differs from the one of [20]: our main result is the interior sphere property of attainable sets, for which we need no controllability assumption of type (46). Then, applying such a property together with assumption (46), we can deduce the semiconcavity of $V_{\mathcal{K}}$ (which in turn yields the interior sphere property of controllable sets).

It is easy to see that, without assumption (46), the interior sphere property may fail for $\mathcal{R}(\mathcal{K} ; t)$. For example, let $u_{0} \in \mathbb{R}^{n}, n \geq 2$, be any vector of norm 2 , and consider the control system $\dot{y}(t)=u(t)$ with $u(t) \in U:=u_{0}+\bar{B}$ and $\mathcal{K}=\{0\}$. Then,

has the interior sphere property, unlike the star-shaped set

$$
\mathcal{A}(\mathcal{K} ; t)=t U=\bar{B}_{t}\left(t u_{0}\right)
$$

$$
\mathcal{R}(\mathcal{K} ; t)=\left\{s u: 0 \leq s \leq t, u \in u_{0}+\bar{B}\right\} .
$$

\section{APPENDIX}

Let $K \subset \mathbb{R}^{n}$ be a closed set and $x \in \partial K$. We recall that a vector $p \in \mathbb{R}^{n}$ is called a proximal normal to $K$ at $x$ if

$$
\exists \lambda>0 \quad \text { such that } \quad d_{K}(x+\lambda p)=\lambda|p|
$$

In this case, one has that $\bar{B}\left(x+\lambda^{\prime} p, \lambda^{\prime}|p|\right) \cap K=\{x\}$ for every $\lambda^{\prime} \in(0, \lambda)$. We denote by $N_{K}^{\text {prox }}(x)$ the cone of all proximal normals to $K$ at $x$. We note that, for any $x \in \partial K$,

$$
N_{K}^{\text {prox }}(x) \subset N_{K}(x),
$$

whilst the opposite inclusion is false, in general.

Proposition 5.6. Let $F: \mathbb{R}^{n} \rightsquigarrow \mathbb{R}^{n}$ be an upper semicontinuous set-valued map with compact convex values, and let $\alpha>0$. Then, the following properties are equivalent:

(a) for all $x \in K$ and $p \in N_{K}(x)$ there exists $v \in F(x)$ such that $\langle p, v\rangle \geq \alpha|p|$;

(b) for all $x \in K$ and $p \in N_{K}^{\text {prox }}(x)$ there exists $v \in F(x)$ such that $\langle p, v\rangle \geq \alpha|p|$.

Proof. Since the implication $(\mathrm{a}) \Rightarrow(\mathrm{b})$ follows from (53), we need only prove the opposite implication (b) $\Rightarrow(\mathrm{a})$. Incidentally, we note this fact is related to the approximation property in [19], 6.18(a).

Let a point $x_{0} \in \partial K$ and a vector $p_{0} \in N_{K}\left(x_{0}\right)$ be fixed, with $\left|p_{0}\right|=1$. For any $\lambda>0$, let $x_{\lambda} \in K$ be such that

We claim that

$$
\left|x_{\lambda}-\left(x_{0}+\lambda p_{0}\right)\right|=d\left(x_{0}+\lambda p_{0}, K\right)
$$

$$
\frac{x_{\lambda}-x_{0}}{\lambda} \longrightarrow 0 \quad \text { as } \quad \lambda \downarrow 0 \text {. }
$$

Indeed, suppose $\lambda_{i} \downarrow 0$, let $w_{\lambda}:=\left(x_{\lambda}-x_{0}\right) / \lambda$, and observe that, in view of $(54),\left|w_{\lambda}\right| \leq 1+\lambda$. Then, up to extracting a subsequence, $w_{\lambda_{i}}$ will converge to some limit as $i \rightarrow \infty$, say to $w_{0}$. Also, $w_{0} \in T_{K}\left(x_{0}\right)$ owing to $(3)$, 
and so $\left\langle p_{0}, w_{0}\right\rangle \leq 0$ by definition. Since, again by (54), $\left|w_{\lambda_{i}}-p_{0}\right|^{2} \leq 1$, we have that $\left|w_{\lambda_{i}}\right|^{2} \leq 2\left\langle p_{0}, w_{\lambda_{i}}\right\rangle$. In the limit as $i \rightarrow \infty$, this implies that $w_{0}=0$. Since this is true for any sequence $\lambda_{i} \downarrow 0$, our claim is proved.

Now, let us note that $x_{0}+\lambda p_{0}-x_{\lambda} \in N_{K}^{\text {prox }}\left(x_{\lambda}\right)$. Therefore, (b) guarantees that, for some $v_{\lambda} \in F\left(x_{\lambda}\right)$,

$$
\left\langle x_{0}+\lambda p_{0}-x_{\lambda}, v_{\lambda}\right\rangle \geq \alpha\left|x_{0}+\lambda p_{0}-x_{\lambda}\right| .
$$

Since $F$ is usc, we conclude that, for some sequence $\lambda_{i} \downarrow 0, v_{\lambda_{i}}$ will converge to some limit in $F\left(x_{0}\right)$, say $v_{0}$. Moreover, dividing by $\lambda_{i}$ both sides of (56), we obtain

$$
\left\langle p_{0}-\frac{x_{\lambda_{i}}-x_{0}}{\lambda_{i}}, v_{\lambda_{i}}\right\rangle \geq \alpha\left|p_{0}-\frac{x_{\lambda_{i}}-x_{0}}{\lambda_{i}}\right| .
$$

Hereby, (55) implies that $\left\langle p_{0}, v_{0}\right\rangle \geq \alpha\left|p_{0}\right|$, as required.

\section{REFERENCES}

[1] J.-P. Aubin, A. Cellina, Differential Inclusions. Springer-Verlag, Berlin (1984).

[2] J.-P. Aubin, H. Frankowska, Set-Valued Analysis. Birkhäuser, Boston (1990).

[3] M. Bardi, I. Capuzzo Dolcetta, Optimal control and viscosity solutions of Hamilton-Jacobi equations. Birkhäuser, Boston (1997).

[4] M. Bardi, M. Falcone, An approximation scheme for the minimum time function. SIAM J. Control Optim. 28 (1990) 950-965.

[5] A. Bressan, On two conjectures by Hájek. Funkcial. Ekvac. 23 (1980) 221-227.

[6] P. Cannarsa, P. Cardaliaguet, Perimeter estimates for the reachable set of control problems. J. Convex. Anal. (to appear).

[7] P. Cannarsa, C. Pignotti, C. Sinestrari, Semiconcavity for optimal control problems with exit time. Discrete Contin. Dynam. Syst. 6 (2000) 975-997.

[8] P. Cannarsa, C. Sinestrari, Convexity properties of the minimum time function. Calc. Var. 3 (1995) 273-298.

[9] P. Cannarsa, C. Sinestrari, Semiconcave functions, Hamilton-Jacobi equations and optimal control. Birkhäuser, Boston (2004).

[10] F.H. Clarke, Optimization and nonsmooth analysis. Wiley, New York (1983).

[11] R. Conti, Processi di controllo lineari in $\mathbb{R}^{n}$. Quad. Unione Mat. Italiana 30, Pitagora, Bologna (1985).

[12] M.C. Delfour, J.-P. Zolésio, Shape analysis via oriented distance functions. J. Funct. Anal. 123 (1994) 129-201.

[13] H. Frankowska, B. Kas̀kosz, Linearization and boundary trajectories of nonsmooth control systems. Canad. J. Math. 40 (1988) 589-609.

[14] H. Hermes, J.P. LaSalle, Functional analysis and time optimal control. Academic Press, New York (1969).

[15] E.B. Lee, L. Markus, Foundations of optimal control theory. John Wiley \& Sons Inc., New York (1967).

[16] S. Lojasiewicz Jr., A. Pliś, R. Suarez, Necessary conditions for a nonlinear control system. J. Differ. Equ., 59, 257-265.

[17] N.N. Petrov, On the Bellman function for the time-optimal process problem. J. Appl. Math. Mech. 34 (1970) 785-791.

[18] A. Pliś, Accessible sets in control theory. Int. Conf. on Diff. Eqs., Academic Press (1975) 646-650.

[19] R.T. Rockafellar, R.J.-B. Wets, Variational analysis. Springer-Verlag, Berlin (1998).

[20] C. Sinestrari, Semiconcavity of the value function for exit time problems with nonsmooth target. Communications on Pure and Applied Analysis. Commun. Pure Appl. Anal. 3 (2004) 757-774.

[21] V.M. Veliov, Lipschitz continuity of the value function in optimal control. J. Optim. Theory Appl. 94 (1997) 335-363.

[22] P. Wolenski, Y. Zhuang, Proximal analysis and the minimal time function. SIAM J. Control Optim. 36 (1998) 10481072. 